\title{
Behaviour of tracer diffusion in simple atmospheric boundary layer models
}

\author{
P. S. Anderson and S. J.-B. Bauguitte \\ British Antarctic Survey, Madingley Road, Cambridge, CB3 0ET, UK \\ Received: 15 November 2006 - Published in Atmos. Chem. Phys. Discuss.: 13 December 2006 \\ Revised: 20 September 2007 - Accepted: 21 September 2007 - Published: 8 October 2007
}

\begin{abstract}
D profiles and time series from an idealised atmospheric boundary layer model are presented, which show agreement with boundary layer measurements of polar $\mathrm{NO}_{\mathrm{x}}$. Diffusion models are increasingly being used as the framework for studying tropospheric air chemistry dynamics. Models based on standard boundary layer diffusivity profiles have an intrinsic behaviour that is not necessarily intuitive, due to the variation of turbulent diffusivity with height. The simple model presented captures the essence of the evolution of a trace gas released at the surface, and thereby provides both a programming and a conceptual tool in the analysis of observed trace gas evolution. A time scale inherent in the model can be tuned by fitting model time series to observations. This scale is then applicable to the more physically simple but chemically complex zeroth order or box models of chemical interactions.
\end{abstract}

\section{Introduction}

Recent polar air chemistry field campaigns have highlighted the need to integrate air chemistry, boundary layer physics, snow chemistry and snow physics in order to understand the behaviour of trace gas evolution in the lower troposphere. The large investment required to equip a site with both micro-meteorological equipment and a suitable air chemistry facility confines most contemporary studies to existing static research stations, such as (from south to north), South Pole, Dome C, Halley, Greenland Summit, Barrow and Alert

Correspondence to: P. S. Anderson

(philip.s.anderson@bas.ac.uk)
(Davis et al., 2004; Jones, et al., 2007 ${ }^{1}$; Dibb and Jaffrezo, 1997). Inter-site comparisons and contrasts can be made, given the differences in latitude, altitude and maritime influences. King et al. (2006) note that Halley and Dome C experience similar top of the atmosphere solar forcing due to their latitudinal similarities yet they still experience significantly different climatologies due to altitude differences (and thence temperature climatology differences) allowing a difference in the partition of the sensible and latent heat fluxes. Inter-site climatological difference must be taken into account when assessing the difference in the chemical behaviour of the atmosphere.

The physical dynamics of a system can occasionally be the cause of rapidly varying or extremes in chemical signature in the environment (Jones et al., 2006). Frontal events bring air masses with differing histories to the sampling inlet, and the frontal interface may be remarkably narrow. Identifying when an observed tracer variation is due to chemical dynamics or due to physical advection requires either spatial measurements or a reliable boundary layer model of the atmosphere. In polar studies, cost often preclude such luxuries as the former. The onus is therefore on the intuitive skill of the researcher to tease out the truth from the message, and for polar environmental studies, these skills need to be multidisciplinary.

When a perfect model of a system is unavailable, conceptual models become increasingly vital to assist our interpretation of experimental data. Conceptual models are useful in any scientific endeavour, and the exchange of such concepts

\footnotetext{
${ }^{1}$ Jones, A. E., Wolff, E. W., Salmon, R. A., et al.: Chemistry of the Antarctic Boundary Layer and the Interface with Snow: An overview of the CHABLIS campaign, Atmos. Chem. Phys. Discuss., in preparation, 2007.
} 
between different schools often generate their own insights. For the polar boundary layer physics and chemistry community, the interchange of ideas is proving increasingly fruitful. The behaviour of the idealised boundary layer diffusion scheme is both simple enough to envisage, whilst affording some level of accuracy, especially during the polar summer when the atmosphere is relatively well mixed by wind shear or convection. The model is likely to be unsuitable for studying trace gas transfer within the very stable boundary layer, however, due to significant complexities that are not captured by the simple diffusive scheme (Mahrt, 1998). Stability can significantly reduce mixing by suppressing turbulent exchange, and such conditions are ubiquitous during the polar winter over shallow slope topography.

Direct experience of diffusion is limited to approximately linear systems, such as the flow of heat through a conductor or the percolation of cooking smells through the house. In an exact linear diffusive system the resulting flux is directly proportional to a gradient, with the diffusivity being the constant of proportionality. In contrast, the classical atmospheric boundary layer diffusive model incorporates a variable diffusivity, one that is a function of height. The simplest approximation for $K(z)$ is to extend the known surface layer diffusivity which is linearly proportional to height. When used in 1-D models, this form of diffusivity generates profiles of wind, temperature, tracer etc, that evolve towards straight lines when plotted against $\ln (z)$ and are known as "log-linear" profiles.

In both the real world and 1-D diffusion models there is usually some upper level where the diffusivity becomes zero. This level defines the top of the boundary layer, and may be regarded as the upper extent of a containment vessel for surface processes. Intuition would indicate that the depth of the boundary layer and the rate of exchange between the surface and the rest of the atmosphere would be the main factors governing the shape of the resulting profiles. This detracts from two other significant factors; the variation of diffusivity with height, and the loss of the tracer out of the system. As will be shown below, for certain cases, the depth of the boundary layer is irrelevant in determining the trace gas profile or the time series near the surface.

The model used to generate the profiles presented here is the simplest physical description that represents a real atmospheric boundary layer. The modelled concentration of a virtual tracer in the near surface layer agree well with real measurements made at the Halley research station in Antarctica. The model is relatively insensitive to physical parameters such as wind speed or boundary layer depth, compared to the sensitivity to changes in typical values of the tracer decay (or loss) term, $\tau$. The loss term can be tuned to real time series, and hence provides the effective time scale that is relevant for zeroth order box models of realistically complex chemistry.

The model equations and boundary conditions are described in the first part of the Methods section. The validity of using global radiation data as a proxy for trace flux at the surface is considered, given the comparison of model output with measurements of $\mathrm{NO}_{\mathrm{x}}$ later in the paper. Certain practical aspects of solving the equations are discussed. Finally, the averaging and function fitting techniques used to compare $\mathrm{NO}_{\mathrm{x}}$ data with the model time series are presented.

The Results section presents typical profiles and time series of tracer concentration that are solutions to the diffusion equation, along with their sensitivity to changing such physical parameters as boundary layer depth, and roughness length.

The Discussion section describes the time scales for which the boundary layer depth is not important, and some consequences of log-linear profiles. The Discussion ends with possible explanations for asymmetry in the observed $\mathrm{NO}_{\mathrm{x}}$ data time series.

\section{Method}

\subsection{Model description and boundary and initial conditions}

The model is a one-dimensional time-dependent diffusion scheme with height dependent diffusivity, $K(z)$.

The diffusion and loss model are described by

$$
\frac{d C}{d t}=\frac{d}{d z}\left(K \frac{d C}{d z}\right)-\frac{C}{\tau}
$$

where $t$ is time, $z$ is height, $K$ the local diffusivity and $\tau$ a loss term which defines the rate of decay of $C$ in a gradientfree environment. A Crank-Nicholson scheme with $N$ levels is used to solve each time step of the diffusion term in Eq. (1), then the loss term was subtracted from the profile. The model levels were spaced logarithmically such that the height, $z_{n}$, of level $n(n=0: N-1)$ was given by:

$$
\begin{aligned}
& z_{n}=z_{0} e^{n . \Delta z} \\
& \Delta z=\frac{\ln (h)-\ln \left(z_{0}\right)}{50}
\end{aligned}
$$

where $h$ is the prescribed height of the upper level. For these studies the profile and time series results did not vary for $N$ above $\sim 50$. The model time step was $5 \mathrm{~min}$.

The diffusivity, $K(z)$ is given by

$K(z)=0.4 z u_{*}$

where the friction velocity, $u_{*}$, is derived from the near surface form of the neutral boundary layer equation, that is:

$\frac{0.4 U}{u_{*}}=\ln \left(\frac{z}{z_{0}}\right)$

where $U$ is the wind speed at height $z$. The value 0.4 is the von Karman constant, usually represented by $\kappa$, the Greek letter kappa. I have used 0.4 in equations to avoid confusion with the diffusivity terms, such as $K$.

$K$ is linearly dependent upon $z$ which leads to $K=0$ at the surface. This is obviously unrealistic and the model assumes 
that this form of $K$ is only valid fo $z \geq z_{0}$, in accord with physical models of surface layer stress and heat flux.

The model was initialised with zero concentration at all levels, and then spun up for five days before the profile or time series were extracted.

\subsection{Forcing term}

There is no flux across the upper boundary, that is, the gradient is forced to be zero. The height of this upper level, $h$, is prescribed and defines the top of the turbulent boundary layer.

The lower boundary condition assumes a flux of trace gas which is proportional to the time dependent global radiation, $G(t)$.

$$
\left.K \frac{d C}{d z}\right|_{z=z 0} \propto G(t)
$$

where $C$ is the concentration. This is a central aspect of the time dependent forcing terms of the model, and therefore requires some discussion to justify the validity of the links in the chain of assumptions. In brief these assumptions are:

1. Flux of tracer out of the surface is instantaneous following generation within the snow pack: snow diffusion is rapid and/or distances are small.

2. In-snow generation of tracer is limited by (and therefore proportional to) the column integrated in-snow actinic flux for nitrate, $\int j \mathrm{X} \mathrm{d} z$ : the reduction of source chemical (e.g. nitrate for $\mathrm{NO}_{\mathrm{x}}$ production) is neglected.

3. $\int j \mathrm{X} \mathrm{d} z$ is proportional to the global shortwave irradiance, $G$.

The assumption of rapid diffusion is supported by snow model results from Lee-Taylor and Madronich (2002) showing the bulk of actinic activity to be within $10 \mathrm{~cm}$ of the surface, along with measurements of snow pack diffusivity of order $10^{-5} \mathrm{~m}^{2} \mathrm{~s}^{-1}$ (Trudinger et al., 1997), giving diffusion time scales of the order of minutes (Wolff et al., 2002).

Wolff et al. (2002) report on model results that imply that, at least for photolysis of nitrate, depletion is only significant in the upper snow layers when accumulation is small $\left(27 \mathrm{~kg} \mathrm{~m}^{-2} \mathrm{yr}^{-1}\right)$ such as may occur deep in the Antarctic interior.

The proportionality between surface irradiance and column integrated actinic flux for nitrate, $\mathrm{NO}_{3}^{-}$, is discussed by Lee-Taylor and Madronich (2002). Using a coupled snow and atmospheric radiative transfer model, their study shows that for moderate solar zenith angles $\left(60^{\circ}\right)$ there is a linear relation between $\int j \mathrm{NO}_{3}^{-} \mathrm{d} z$ and the irradiance for $\mathrm{NO}_{2}$ channel, $j \mathrm{NO}_{2} \downarrow$. Similarly, Wolff calculates the actinic photolysis frequencies of nitrate in snow, which approximate to a gaussian wavelength function with a mean wavelength at
$320 \mathrm{~nm}$ and width $\sim 8 \mathrm{~nm}$ (estimated from Wolff et al., 2002). That is, in the formal photolytic equation:

$\frac{d[\mathrm{X}]}{d t}=-[X] \cdot \int \sigma_{X}(\lambda) \phi(\lambda) E(\lambda) d \lambda$

where $X$ is the source substance, $\sigma_{x}$ the absorption cross section, $\phi$ the quantum yield and $E$ the spectral actinic flux, the function under the integral is approximately:

$A \exp \left(\frac{(\lambda-\mu)^{2}}{2 \omega^{2}}\right)$

where $\mu=320 \mathrm{~nm}$ and $\omega=8 \mathrm{~nm}$. The sensitivity of this frequency band to zenith angle can be assessed by convolving Eq. (7) with surface irradiance spectra derived from a radiation transfer model (e.g. TUV from (http://cprm.acd. ucar.edu/Models/TUV/Interactive_TUV/). This shows that, despite the known peak in the nitrate action spectrum $\left(\sigma_{x}\right.$, $\phi)$ at UV wavelengths, the overall $\left(\sigma_{x} \phi \mathrm{E}\right)$ integral is linearly dependant upon total irradiance, at least for zenith angles less than $80^{\circ}$. Given that $j \mathrm{NO}_{2} \downarrow$ also peaks between $350-400 \mathrm{~nm}$, this explains the near linear agreement between $\int j \mathrm{NO}_{3}^{-} \mathrm{d} z$ and $j \mathrm{NO}_{2} \downarrow$ found by Lee-Taylor and Madronich.

Finally, comparing measured clear sky $G$ at $76^{\circ} \mathrm{S}$ for 14 January 2005 and the convolved TUV spectra for identical latitude, altitude and date over a diurnal cycle confirms the approximate linearity of the transfer function, $G \rightarrow \int j \mathrm{NO}_{3}^{-} \mathrm{d} z$ for this period with mean error of $\sim 20 \%$ assuming a linear approximation that is forced to pass through the origin. Error reduces to $1.2 \%$ if a quadratic approximation is used, which captures the reduction in $\int j \mathrm{NO}_{3}^{-} \mathrm{d} z$ at low zenith angles.

\subsection{Model sensitivity}

The model generates a time series of concentration profiles, $C(t, z)$ from which profiles at a given time or a time series at a given height can be extracted. The significant prognostic physical parameters in the model were domain boundaries, $h$ and $z_{0}$, and the wind speed, $U$, from which $u_{*}$ and hence $K(z)$ were derived. A measure of the general behaviour of the model is given by the time lag, $\Delta t$, between maximum $G(t)$ and maximum $C(z, t)$ at a given height. $\Delta t$ can then be presented graphically as a function of the loss time constant, $\tau$. The sensitivity of the model to changes in $h, z_{0}$ and $U$ is then shown as effects on behaviour of $\Delta t(\tau)$.

\section{$2.4 \mathrm{NO}, \mathrm{NO}_{2}$ and $\mathrm{NO}_{\mathrm{x}}$ measurements}

Near surface measurements of $\mathrm{NO}$ and $\mathrm{NO}_{2}$ from an Antarctica station were available as part of the CHABLIS air chemistry campaign and were used to test the validity of the model. The measurements were made in the Clean Air Sector Laboratory (CASLab) at Halley, situated at $76^{\circ} \mathrm{S} 26^{\circ} \mathrm{W}$ 

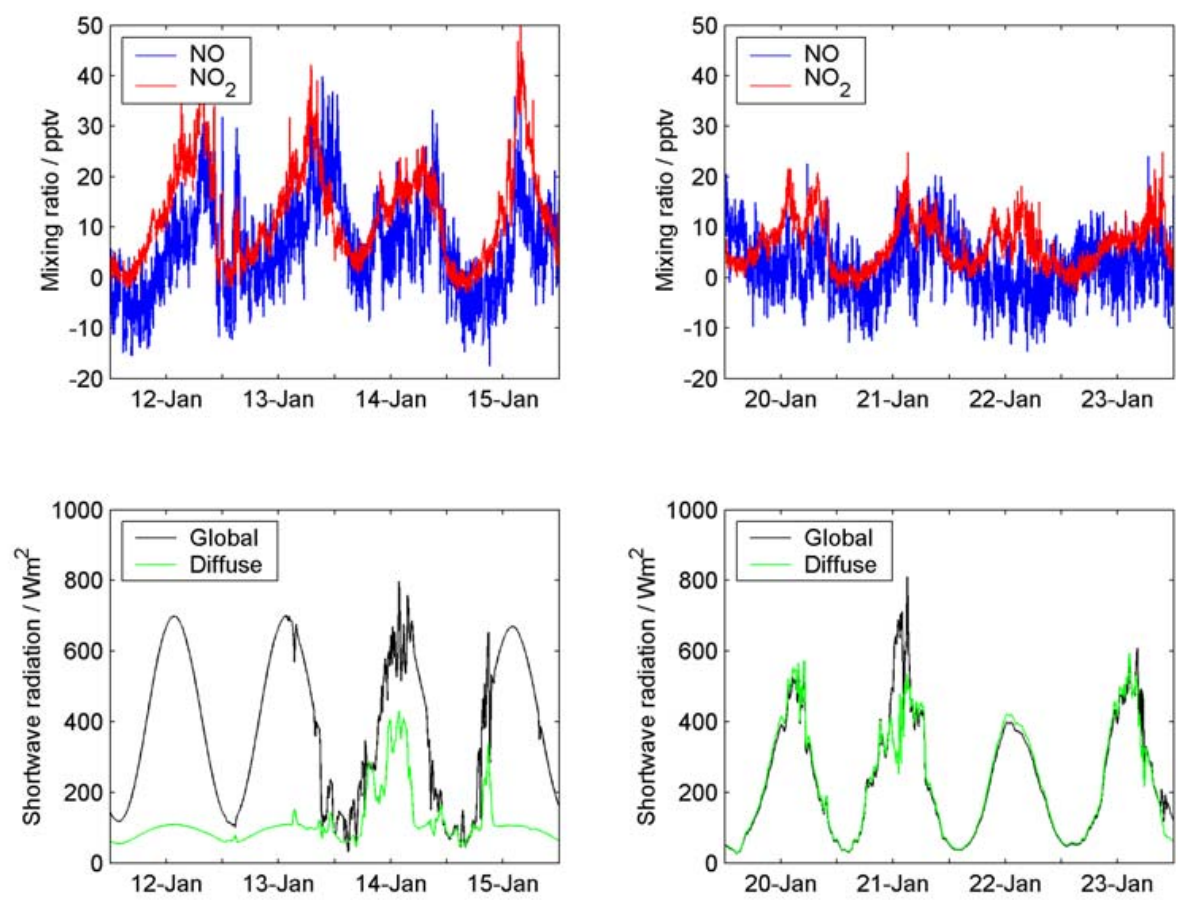

Fig. 1. Time series of $\mathrm{NO}$ and $\mathrm{NO}_{2}$ during January 2005, compared to co-temporal data of solar radiation. The left hand panels are for days when there is little or no cloud cover, as indicated by the difference in the global and diffuse radiation series. The right hand panels are for cloudy days, when global and diffuse radiation are equal.

Table 1. Coefficients of the harmonic fitted curves shown in Fig. 2 and the loss term fitting the observed lag.

\begin{tabular}{|c|c|c|c|c|c|c|}
\hline & Sunny & & & Cloudy & & \\
\hline $\begin{array}{c}\text { name } \\
\mathrm{a}_{0} \text { (mean) }\end{array}$ & $\begin{array}{l}\text { value } \\
14.47\end{array}$ & Max w.r.t LAN & $\tau$ & $\begin{array}{c}\text { value } \\
6.93\end{array}$ & Max w.r.t LAN & $\tau$ \\
\hline $\begin{array}{l}\mathrm{a}_{s} \\
\mathrm{a}_{c}\end{array}$ & $\begin{array}{c}-14.63 \\
-3.70\end{array}$ & 3.32 & 2.44 & $\begin{array}{l}-5.31 \\
-2.62\end{array}$ & 2.52 & 1.80 \\
\hline $\begin{array}{l}\mathrm{b}_{s} \\
\mathrm{~b}_{c}\end{array}$ & $\begin{array}{l}-2.25 \\
-2.29\end{array}$ & 4.37 & & $\begin{array}{l}-0.27 \\
-0.19\end{array}$ & 2.53 & \\
\hline
\end{tabular}

on the Brunt Ice Shelf. A description of the relevant instrumentation and site layout is given in Jones et al. (2007) ${ }^{1}$. The post processing and quality control of the $\mathrm{NO} / \mathrm{NO}_{2}$ data are described in Bauguitte et al. (2007) ${ }^{2}$. Halley's Clean Air Sector is equipped with a suite of micro-meteorological instruments, from which realistic estimates of the model's prognostic physical parameters have been taken.

Two case study periods of $\mathrm{NO}$ and $\mathrm{NO}_{2}$ time series were chosen from the full data set (Fig. 1), both four days in length but differing in the amount of cloud cover. The days from 12

\footnotetext{
${ }^{2}$ Bauguitte, S. J.-B., Bloss, W. J., Evans, M. E., et al.: An overview of multi-seasonal $\mathrm{NO}_{\mathrm{x}}$ measurements during the CHABLIS campaign: Can sources and sinks estimates unravel observed diurnal cycles?, Atmos. Chem. Phys. Discuss., in preparation, 2007.
}

to 15 January 2005 are mostly cloud free, whilst from 20 to 23 January are cloudy.

The daily $\mathrm{NO}$ and $\mathrm{NO}_{2}$ were added to give a time series of $\mathrm{NO}_{\mathrm{x}}$, the relevant tracer for studying nitrate photolysis in snow (Wolff et al.). Figure 2 shows the resulting time series, presented as 48 bins of width $30 \mathrm{~min}$, error bars indicating one standard deviation. Overlaid are fitted harmonic functions. The green curve, $A_{G}(t)$ is given by:

$$
A_{G}(t)=a_{0}+a_{s} \sin (2 \pi t / 24)+a_{c} \cos (2 \pi t / 24)
$$

where time is given in hours. For the sunny data (LHS panel, Fig. 2), there is a systematic disagreement between the data and this harmonic function; the data are not symmetrical about the maximum. To highlight this, $A_{R}(t)$ was fitted, 

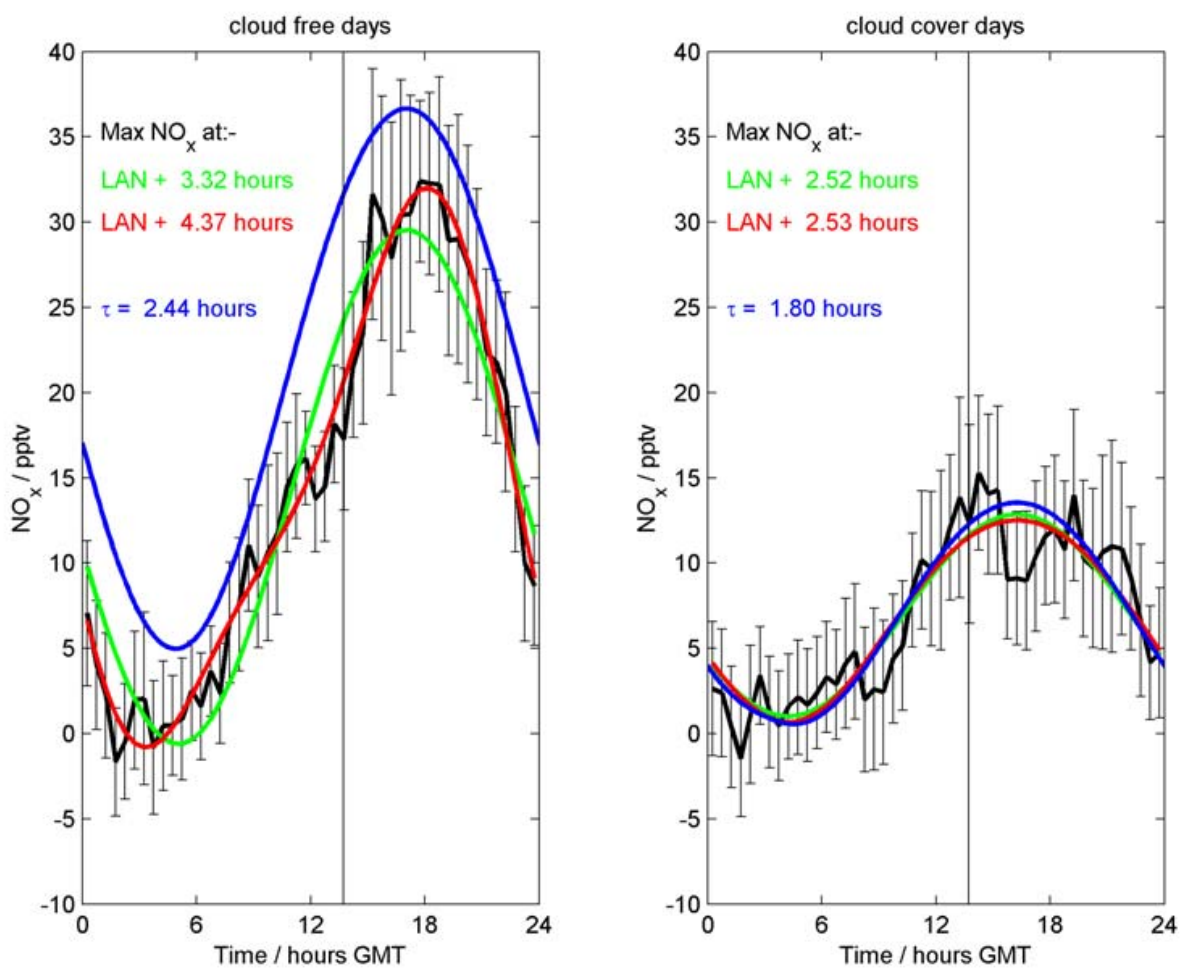

Fig. 2. Daily averaged time series of $\mathrm{NO}_{\mathrm{x}}\left(\mathrm{NO}+\mathrm{NO}_{2}\right)$ for the same data as in Fig. 1, with the cloud free data on the left and the cloud cover data on the right. Overlaid on each plot are three functions fitted to the binned data by least squares. In green and red are harmonic functions; the green function uses a single harmonic as per Eq. (8), the red function has first and second harmonics as per Eq. (9). For the cloud free days, the red curve is a significantly better fit, implying an asymmetry to the $\mathrm{NO}_{\mathrm{x}}$ time series. The harmonic functions for the cloud cover data are equivalent. The blue curves show diffusion model fits, where $\tau$ has been tuned to give the same post-LAN maximum as the single harmonic function. For the cloud free data there is a significant mean discrepancy between the diffusion model and the data, whilst for the cloud cover days, this offset is absent, implying some real effect, and not an artefact of the instrument.

shown in red, which included both first and second harmonics, that is:

$$
\begin{aligned}
A_{R}(t) & =a_{0}+a_{s} \sin (2 \pi t / 24)+a_{c} \cos (2 \pi t / 24) \\
& +b_{s} \sin (4 \pi t / 24)+b_{c} \cos (4 \pi t / 24)
\end{aligned}
$$

\subsection{Model validation via tuning}

To test the validity of the diffusion/loss model, the phase offset between model and measured $\mathrm{NO}_{\mathrm{x}}$ maximum were compared. Absolute values of $\mathrm{NO}_{\mathrm{x}}$ concentration are not produced by the model, due to uncertainties in the chain of efficiencies from $G$ to surface $\mathrm{NO}_{\mathrm{x}}$ flux: the comparison of phase shift and relative variance, however, are not affected by this limitation. The sole free parameter used to tune model was the loss time constant, $\tau$, from whence asymmetry and relative variance could be compared.

Tuning of $\tau$ was achieved by the unconstrained nonlinear minimization method intrinsic to the Matlab? fminsearch.m function. The model output, $C(z, t)$, at $z=4 \mathrm{~m}$ was taken to compare with the $\mathrm{NO}_{\mathrm{x}}$ data; this time series is referred to as $C_{4}(t)$. The values of the coefficients resulting from fitting
$A_{G}$ and $A_{R}$ to both the sunny and cloudy $\mathrm{NO}_{\mathrm{x}}$ data of Fig. 2 are summarised in Table 1.

The model output is in arbitrary units; once $\tau$ was tuned to generate the desired $\Delta t, C_{4}(t)$ was scaled to give agreement with the diurnal variation in $\mathrm{NO}_{\mathrm{x}}$. Hence the diurnal variance of $C_{4}(t)$ and $\mathrm{NO}_{\mathrm{x}}$ are identical, and the mean of $C_{4}(t)$ is then an indication of model - data agreement. This technique of matching phase offset is only suitable for sites where there is a significant diurnal variation in $G(t)$.

A fully refined model, where the dependence of surface $\mathrm{NO}_{\mathrm{x}}$ flux on the incident radiation is known, would allow the absolute magnitude of the model concentration to be compared. The present purpose is to indicate how diffusion and loss contribute to the near surface tracer time series.

\section{Results}

\subsection{Model profiles}

Figure 3 shows typical profiles of $C(z, t)$ for different value of the loss constant, $\tau$. Boundary layer depth, $h$, is $50 \mathrm{~m}$, 

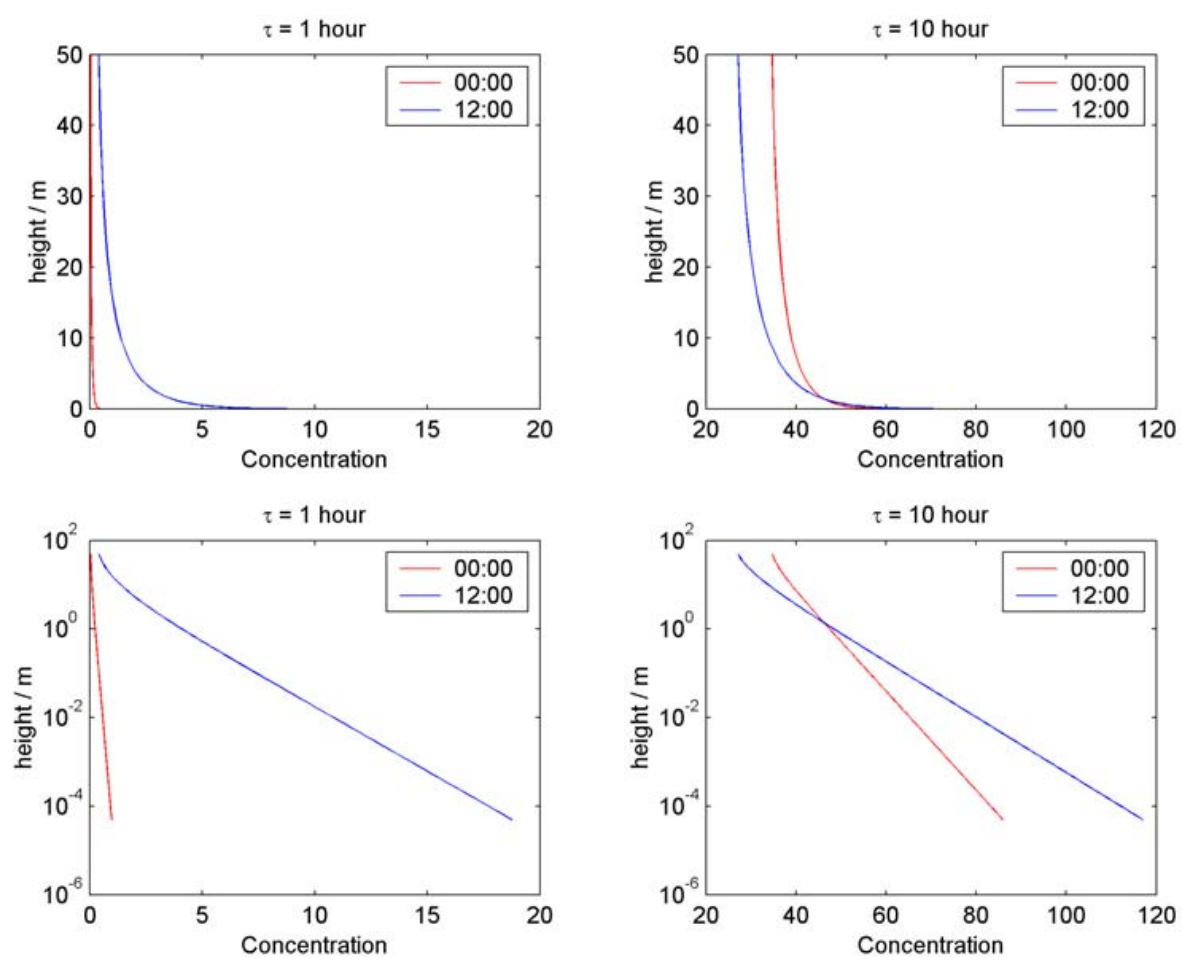

Fig. 3. Profiles of diffusion model concentration at midnight and midday for different values of loss term, $\tau$. The profiles are plotted with both linear and $\log$ height axes. Note the change in scale for the concentration for the different values of $\tau$. $G(t)$ forcing is for 28 October at $76^{\circ}$ South.

roughness length, $z_{0}$ is $5 \times 10^{-5} \mathrm{~m}$ and wind speed, $U$, is $1 \mathrm{~ms}^{-1}$. The left hand panels are midnight and midday profiles for $\tau=1 \mathrm{~h}$, the right hand panels show the same profiles for a ten-fold increase in $\tau$. The upper set of panels show the profile against linear height, whereas the lower set are plotted against $\log _{10} z$. The forcing term, $G(t)$, is appropriate for 28 th October at $76^{\circ} \mathrm{S}$, which includes a few hours of darkness around midnight. This date was chosen to highlight the effect of persistence in surface $\mathrm{NO}_{\mathrm{x}}$ and the shape of $\mathrm{NO}_{\mathrm{x}}(z)$ profiles during darkness.

The upper panels demonstrate the very large gradients in $C(z, t)$ that occur at the surface due to the small values of diffusivity near $z_{0}$. For the larger value of $\tau$, (RHS) there is tracer throughout the model domain, whilst for small $\tau$ (rapid loss) there is almost no tracer at the top. In this latter case, $h$ could be increased indefinitely with no effect on the resulting profiles.

The lower panels, with a logarithmic height axis, highlight the log-linear aspect of the profiles, even when the loss term is large enough for the boundary layer depth to affect $C(z, t)$.

For small $\tau$, almost all tracer has been lost from the domain at midnight, whilst for larger $\tau$, the midnight values aloft exceed the midday values. Only near the surface, $(z<1 \mathrm{~m})$ do the midday values exceed those at midnight. The $\log _{10} \mathrm{z}$-axis plot (lower right) highlights this effect, with the two profiles crossing over.

\subsection{Model time series}

The corresponding time series of concentration at $4 \mathrm{~m}, C_{4}(t)$, for the same parameters as used in the profile examples above are shown in Fig. 4. Three days are presented, showing the model has achieved a repeating cycle for the given repetitive diurnal forcing, and indicating that the spin-up time (5 days) is sufficient. The upper panel is $C_{4}(t)$ for $\tau=1 \mathrm{~h}$, the lower panel for $\tau=10 \mathrm{~h}$. The forcing term, $G(t)$, is indicated in the middle panel. When $\tau$ is large, there is residual tracer at $4 \mathrm{~m}$ following the dark period $(G(t)=0$ at $t=0,1$ etc. $)$, and the mean value is elevated compared to the $\tau$. In both cases there is a lag observed between the maximum in $G(t)$ at Local Apparent Noon (LAN) and the maximum in $C_{4}(t)$, but the lag is greater for larger $\tau$.

\subsection{Sensitivity}

In order to quantify the sensitivity of the model to changes in parameter values, two measures of the behaviour of the model are used:

- $\Delta t$, the time delay in the response of the tracer at $4 \mathrm{~m}$ $C_{4}(t)$ relative to the surface flux

- $R=\sigma C_{4}(t) /<C_{4}(t)>$, the ratio of the standard deviation of the time series to the mean value. 

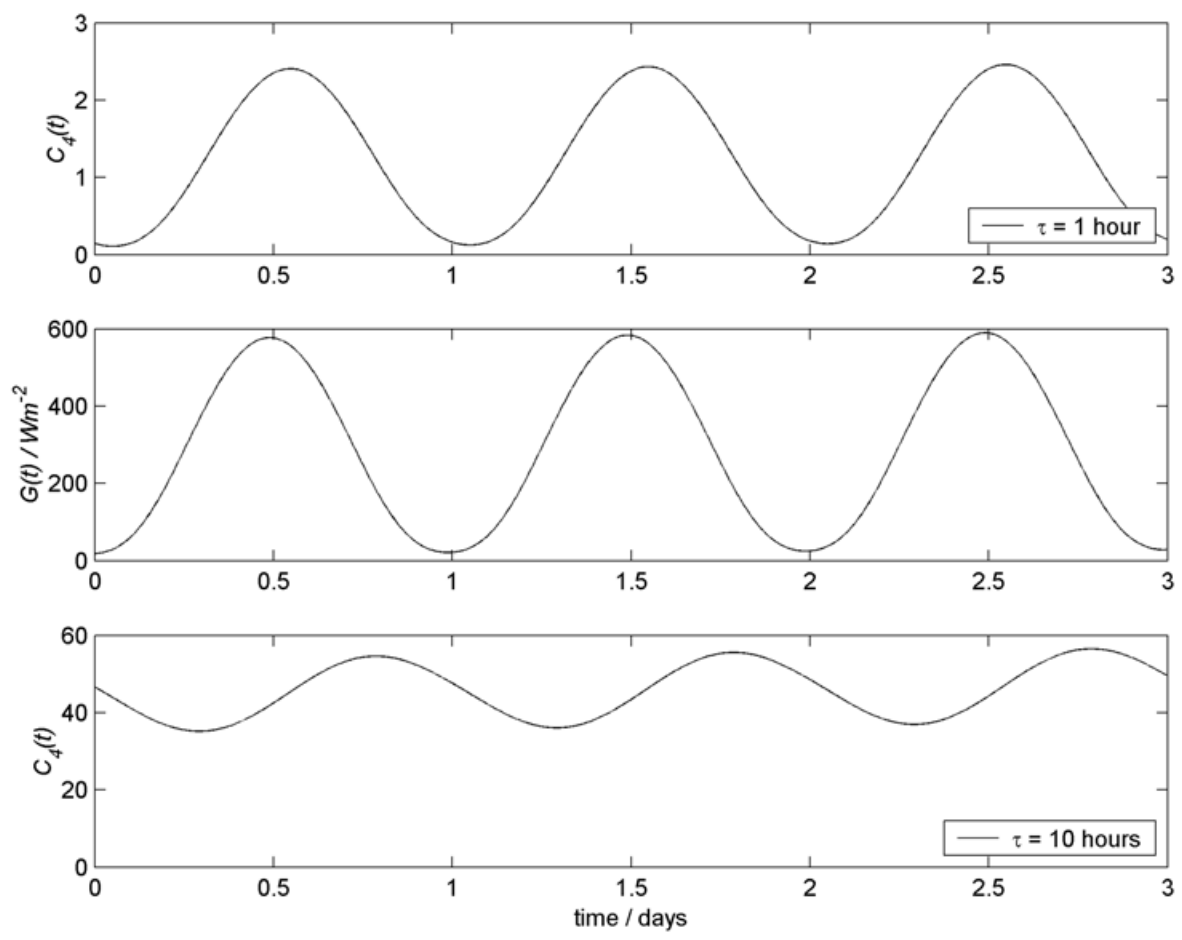

Fig. 4. Time series of diffusion model concentration at $4 \mathrm{~m}$ for the same model runs as Fig. 3. Three days of model output are shown, following five days of spin up; diurnal forcing is shown in the middle panel, and is identical for all model days, including spin up. The similarity of diurnal variation indicates that the model is in a periodic steady state.

The broad scale behaviour of $\Delta t$ and $R$ as a function of the loss term, $\tau$, can be judged from the two time series of $C_{4}(t)$ shown in Fig. 4. For small values of $\tau$ (upper panel) $C_{4}(t)$ is approximately an offset sinusiod function with a minimum of zero and maximum that is twice the mean. The ratio, $R$, for such a function is 0.71 . For large values of $\tau$ (lower panel), the variability in $C_{4}(t)$ is smaller relative to the mean, due to the presence of residual tracer after the dark period.; hence $R$ becomes smaller as $\tau$ increases. The delay in the peak of $C_{4}(t)$ from LAN increases with $\tau$.

Figure 5 shows $R$ and $\Delta t$ as functions of $\tau$ for four different sets of physical parameters. $R(\tau)$ (upper panel) tends to 0.7 as $\tau$ tends to zero, decreasing for increasing $\tau$. $\Delta_{t}(\tau)$ is proportional to $\tau$ for $\tau<\sim 4 \mathrm{~h}$ (lower panel). For larger $\tau$ the gradient decreases, with ever larger $\tau$ only increasing the lag by a small margin. Overlaid on the figure are four model "sets". The "standard" model uses the parameters described in Table 2. The other model runs use the same parameters, but with one parameter altered by a significant (but realistic) amount. These are also given in Table 2.

\subsection{Comparison with $\mathrm{NO}_{\mathrm{x}}$ measurements}

The two time series sets of $\mathrm{NO}$ and $\mathrm{NO}_{2}$ field measurements are present in Fig. 1, along with the co-temporal surface shortwave (solar) radiation. The model forcing, $G(t)$, are taken from smoothed functions of these radiation data. Note
Table 2. Values of internal parameters used to test the sensitivity of the model. The results of these changes are summarised in Fig. 5.

\begin{tabular}{cccc}
\hline Parameter & $\mathrm{h} / \mathrm{m}$ & $\mathrm{U} / \mathrm{ms}^{-1}$ & $\mathrm{z}_{0} / \mathrm{m}$ \\
\hline $\begin{array}{l}\text { standard } \\
\text { change } \mathrm{h} \\
\text { change } \mathrm{U}\end{array}$ & 50 & 1 & $5 \times 10^{-5}$ \\
change $\mathrm{z}_{0}$ & & 5 & \\
\hline
\end{tabular}

that these data are plotted against GMT and mean LAN is offset from GMT noon by 26 degrees, equivalent to $1 \mathrm{~h}, 44 \mathrm{~min}$. The plots have not been corrected for the small additional component of the Equation of Time. Both global and diffuse radiation are shown, global being the sum of diffuse and direct shortwave radiation. On cloudy days there is no direct shortwave radiation and global and diffuse measurements are identical. By contrast on sunny days most shortwave is direct and the diffuse radiation is a small component of the total. An estimate of the effect of the cloud can be judged by comparing time series of these two radiation measurements.

The magnitude of the incident shortwave radiation is reduced by cloud cover, by as much as $40 \%$ in these data, due to light being reflected from the cloud top as shown in Fig. 2. 

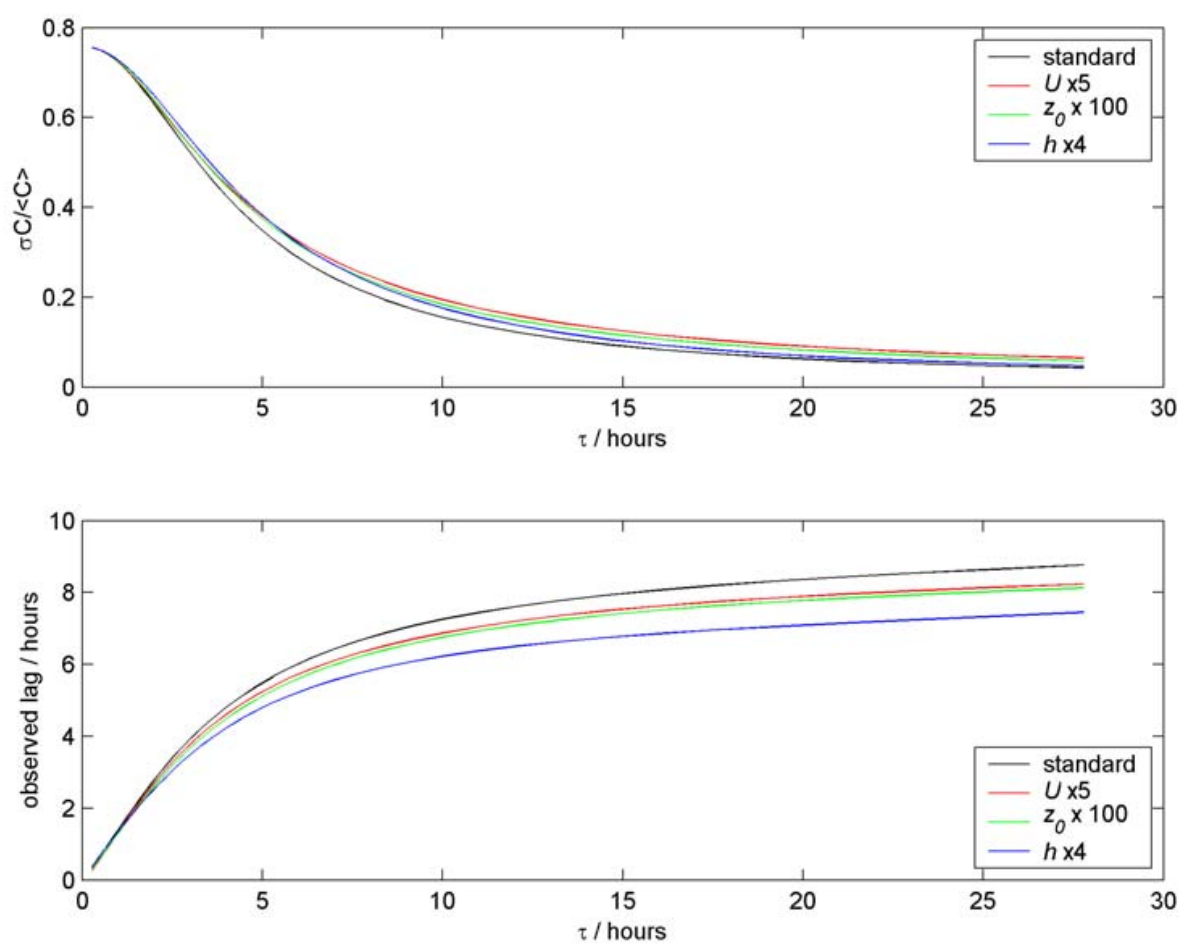

Fig. 5. Diffusion model normalised variance (upper panel) and lag relative to $G(t)$ (lower panel) as a function of loss term, $\tau$. The relative insensitivity of the model to changes in other internal parameters (given in Table 2 ) is indicated by the four curves.

For the cloud free data (LHS panel), there is a systematic disagreement between the data and the first order harmonic function (green) indicating that the data are not symmetrical about the maximum. To emphasis this asymmetry, the second order (red) function fits far better. The difference in the maxima of the two harmonic functions is $\sim 1 \mathrm{~h}$.

In marked contrast to the sunny data, the harmonic curves on the right hand panel for the days of cloud cover are almost identical: the cloudy data are symmetrical about the maximum and there is minimal second harmonic signature in the data.

The final curve in green is $C_{4}(t)$, the output from the model when the loss term, $\tau$, has been tuned to generate the same delay in peak concentration. In both cases (sunny and cloudy data), $\tau$ was tuned to match the delay given by the first order harmonic only. Note that the amplitude of the signature variance has been matched, such that the model variance equals the data variance. An alternative scaling is matching the mean values of the data and the model output. This would be just as valid given the arbitrary units of $C_{4}(z, t)$, and the magnitude of the scaling term is similar, but the model-todata comparison is clearer using the variances.

There is, again, a notable difference between the two case studies. For the sunny data, the model indicates an offset relative to the harmonic fit of about 4 pptv, whereas the cloud data shows a near exact fit. The model offset observed for the sunny days is due to the larger value of $\tau$ required to match the lag maximum concentration.

\section{Discussion}

\subsection{Steady state conditions: the log-lin profile}

Figure 3 shows profiles of tracer for two different loss time scales; $\tau=1 \mathrm{~h}$ and $\tau=10 \mathrm{~h}$. The lower panels show the near linearity of the resulting profiles when plotted using $\log z$. This allows a conceptual picture of the profile for different loss time scales. In the case where $\tau=1 \mathrm{~h}$, there is very little tracer reaching the upper limit to the domain, that is, the top of the boundary layer.

For the linear profiles where $\tau$ is "sufficiently small", extrapolating the linear part of the profile to $C=0$ gives a scale height, $h_{C}$. This may be thought of as an effective trace gas boundary layer depth, analogous to the physical boundary layer depth, $h$. In both cases, the magnitude of a flux has decreased to zero. Multiple runs of the model with different $\tau$ and $u_{*}$ indicate that:

$h_{C} \propto \tau u_{*}$

This is not unexpected, given that $\tau u_{*}$ is a length scale. Of interest is that the constant of proportionality is close to 0.16 , that is, the square of the von Karman constant.

The $\tau u_{*}$ scale is only applicable if $h_{C}<h$, which defines the term "sufficiently small" for $\tau$. If $h_{C}$ as calculated by Eq. (10) is larger than $h$, the depth of the boundary layer will have a significant effect on the profiles of $C(z, t)$, by capping vertical flux aloft. 
An exact log-linear profile under-estimates the total mass of tracer in the column, $M$, by a factor of about two, due to the slight non-linearity at the upper boundary, that is, if a linear profile is fitted to $C(\ln z)$ for near surface $C$ :

$C_{f i t}(z)=a \ln (z)+b$

them $M$ is about twice integral of $C_{f i t}$. The integration of Eq. (11) is

$$
\begin{aligned}
\int_{0}^{h_{C}} C(z) d z & =\int_{0}^{h_{C}} a \ln (z)+b d z \\
& =[a \cdot z \cdot(\ln (z)-1)+b z]_{z_{0}}^{h_{C}}
\end{aligned}
$$

Where $z_{0}$ is the roughness length, and $h_{C}$ is given by $-b / a$. For the small values of $z_{0}$ observed over snow, the $z_{0}$ terms are negligible, and the function is simplifies to

$\int_{0}^{h_{C}} C(z) d z=a \cdot h_{C} \cdot\left(\ln \left(h_{C}\right)-1\right)+b h_{C}$

And hence

$M \approx 2 a \cdot h_{C} \cdot\left(\ln \left(h_{C}\right)-1\right)+b h_{C}$

Knowing the total mass, $M$, and the extinction time scale, $\tau$ allows the surface flux, $F_{s}$, to be estimated. Under steady state conditions, or averaged over a day, the flux will be balanced by the total loss aloft, that is

$F_{s}=M / \tau$

The simple model provides an intuitive guide to whether boundary layer depth is a significant parameter in the dynamics of a surface flux of trace gas, and provides some useful simplifications to the expected profiles.

\subsection{Asymmetry in the $\mathrm{NO}_{\mathrm{x}}$ time series}

The two time series of $\mathrm{NO}_{\mathrm{x}}$ measurements shown in Figs. 1 and 2 differ in a number of aspects:

- the amplitude of $\mathrm{NO}_{\mathrm{x}}$ diurnal variability.

- the asymmetry of the same variability

- the phase shift relative to LAN

- the match between the data and the model.

It may well be that the highly complex underlying chemistry acting to photo-chemically generate and then dissociate the components of $\mathrm{NO}_{\mathrm{x}}$ is different on the two occasions; it is possible that there are differences in the history (trajectories) of the air mass at Halley during the two case studies. It may be that the different albedo, and subsequent e-folding depth of the in-snow actinic flux affects other species of trace gas which are involved in the complex air chemistry of $\mathrm{NO}_{\mathrm{x}}$ decay (Warren, 1982). The above notwithstanding, the model can be used to investigate whether there is any simple meteorological (that is physical) explanation which fits the data. In

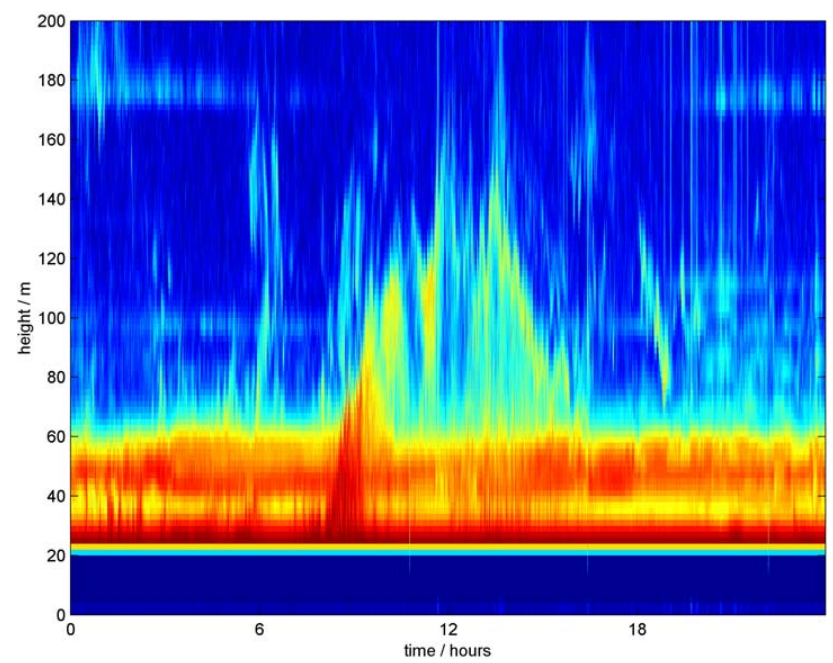

Fig. 6. Acoustic radar echogramme (sodar plot) for the 30 November 2003, indicating the significant increase in mixing height around local apparent noon, starting at 07:00 and decaying by 17:00. Red/orange indicates strong echo from turbulence acting within a temperature gradient, end conversely, blue indicates minimal turbulent mixing. The lower levels are obscured by persistent echoes from nearby buildings.

this spirit of parsimony, the model is perhaps most instructive when data and model disagree. The two case studies of $\mathrm{NO}_{\mathrm{x}}$ time series offer a number of examples.

The significant differences between $\mathrm{NO}_{\mathrm{x}}(t)$ for the two days were a change in the asymmetry in the diurnal variation, the phase shift relative to LAN, the mean and the variance. The significant meteorological difference between the two periods was the amount of cloud cover, with data and model agreeing best on the cloud cover days.

Shortwave radiometers confirm our personal experience that cloud cover reduces the level of insolation reaching the surface, by scattering some of the light back into space. It is therefore to be expected that the magnitude of the peak in $\mathrm{NO}_{\mathrm{x}}$ is smaller on cloudy days. Less obvious is the effect of longwave radiation from the cloud base. Cloud cover over snow will tend to elevate the snow surface temperature until snow, cloud base and intervening air are isothermal (Ambach, 1974). In polar regions, therefore, cloud free conditions may heat the surface (through enhanced insolation) or cool the surface (through reduced thermal radiation), the balance depending on a number of variables, but tending towards heating in mid-summer and cooling at other times. Surface heating can generate convection, which dramatically increases the depth of the boundary layer. As an example of this effect, Fig. 6 shows a sodar plot from Halley for 30 November 2003. Under suitable conditions, the sodar produces a time series of mixing profiles, from which can be inferred the diurnal variation in boundary layer depth, $h(t)$. Including a time dependent boundary layer depth into 

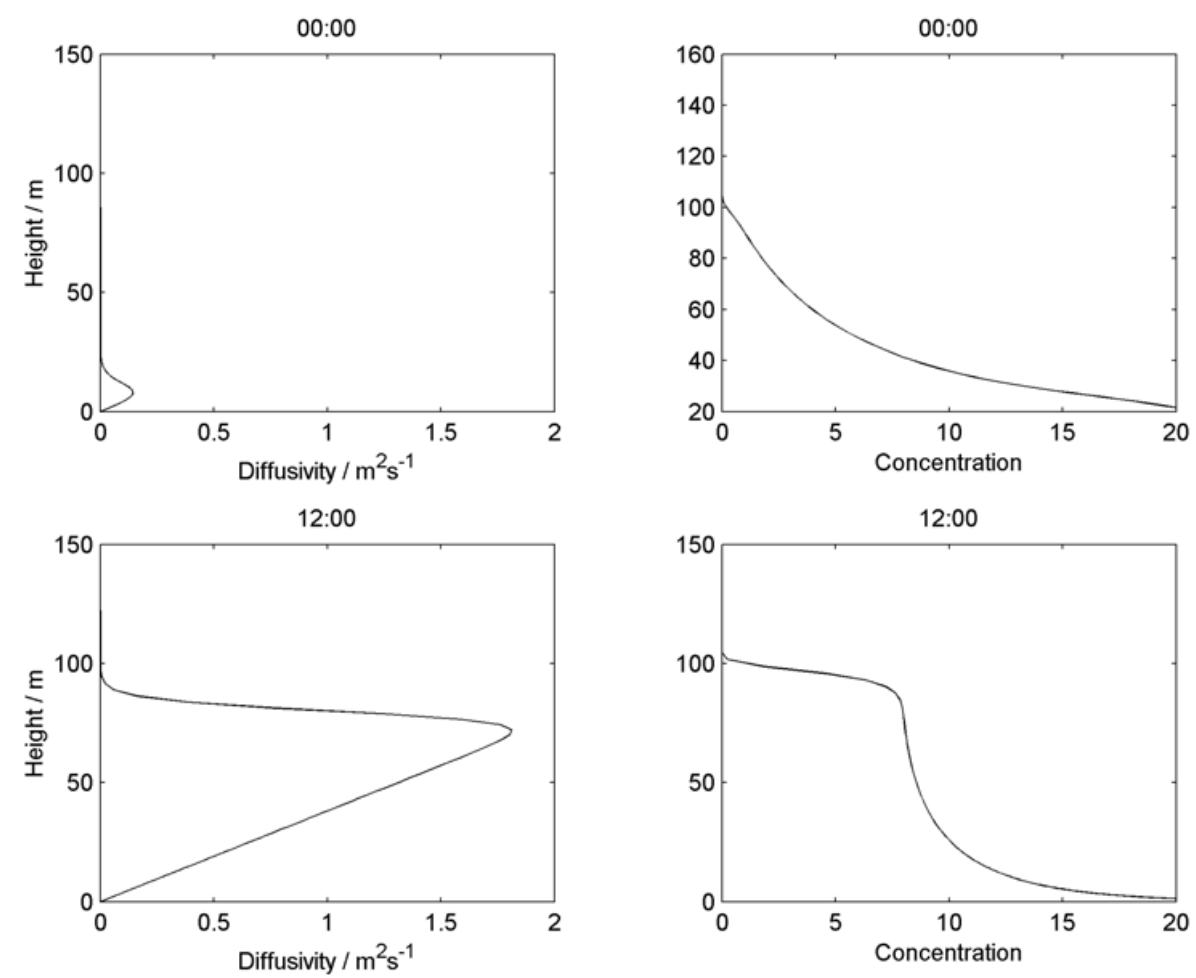

Fig. 7. Four metre tracer concentration profiles $C_{4}(t)$ for a time dependant diffusivity profile, $K(z, t)$. Upper panels are for midnight and lower panels for midday. The diffusivity profiles are shown on the left; near the surface $K(z, t)$ is proportional to height, with a smooth cutoff to zero at the proscribed mixing level height, $h(t)$. The resulting profiles of $C(z, t)$ are shown on the right, with a mixed layer underlying a decaying (non-diffusive) layer. $h(t)$ varies between the 00:00 minimum and the 12:00 maximum as a sinusoid.

the model indicates that a LAN-symmetric increase then decrease in $h$ will generate an asymmetry in measured near surface concentration; not only will there be a lag in the time series as before, but the increase in concentration in the afternoon (observed at $4 \mathrm{~m}$ ) will be slower than the decrease in early evening.

The model implementation used a modified, time dependant, diffusivity profile, $K(z, t)$, whilst maintaining the original 1-D grid; the effect on the modelled profiles was therefore due to a change in diffusivity, not a compression of the boundary layer. $K(z, t)$ is derived by multiplying $K(z)$ in Eq. (1) with a step function at the appropriate height for each time step. The step function is implemented with the convenient $\tanh (\mathrm{x})$ function; hence:

$$
\begin{aligned}
& K(z, t)=0.4 \cdot z \cdot u_{*} \cdot g(t, z) \\
& g(t, z)=1-\tanh (a(z-h(t)))
\end{aligned}
$$

that is, $g(t, z)$ reduces $K$ for $z>h$. The sharpness of the cut off depends upon $a$, a suitable value being $a=0.2$. $h(t)$ will vary smoothly through the day and providing a time-dependent mixing depth. The sodar image of Fig. 6 shows that the actual mixed layer depth can change from less than $20 \mathrm{~m}$ over night to over $100 \mathrm{~m}$ during the middle of the day; the modified model uses these limits for the diurnal variation in $h(t)$.

Figure 7 shows the profiles diffusivity, $K(\mathrm{z}, 00: 00)$ and $K(\mathrm{z}, 12: 00)$ from such a model run, with the resulting con- centration profiles: all other parameters as per the "standard run" in Table 2. At midnight, the $C(z, 00: 00)$ is relatively constant with height for $z<h$, that is within the mixing zone. Above this region there is no mixing, and the profile exhibits a near exponential decay with height. This is the effect of "leaving behind" some of the tracer, as the diffusion reduces to zero at that level, and the tracer equation at these levels being governed solely by the loss term. The higher levels being "left behind" earlier, and have had longer to decay.

At midday, the mixed layer is deeper (as a prognostic), and filled throughout with tracer from the surface. The profile is similar to that for a constant boundary layer depth shown on the right of Fig. 3. Note that the gradient of the diffusivity at the surface is unchanged, and approximates to Eq. (3).

Figure 8 shows the time series of $4 \mathrm{~m}$ concentration, $C_{4}(t)$, that result from varying the mixing depth. The form of $h(t)$ as a sinusoid is shown in the lower right panel with the surface forcing term, $G(t)$, in the upper right; both $G(t)$ and $h(t)$ are symmetric about LAN, but $C_{4}(t)$, shows a slower rate of increase during the afternoon than the subsequent decay in early evening. The effect is indicated by fitting a first order and a second order harmonic function to $C_{4}(t)$ in a similar manner to analysis of the $\mathrm{NO}_{\mathrm{x}}$ data above. The difference in the maxima of the two functions is $0.98 \mathrm{~h}$. 

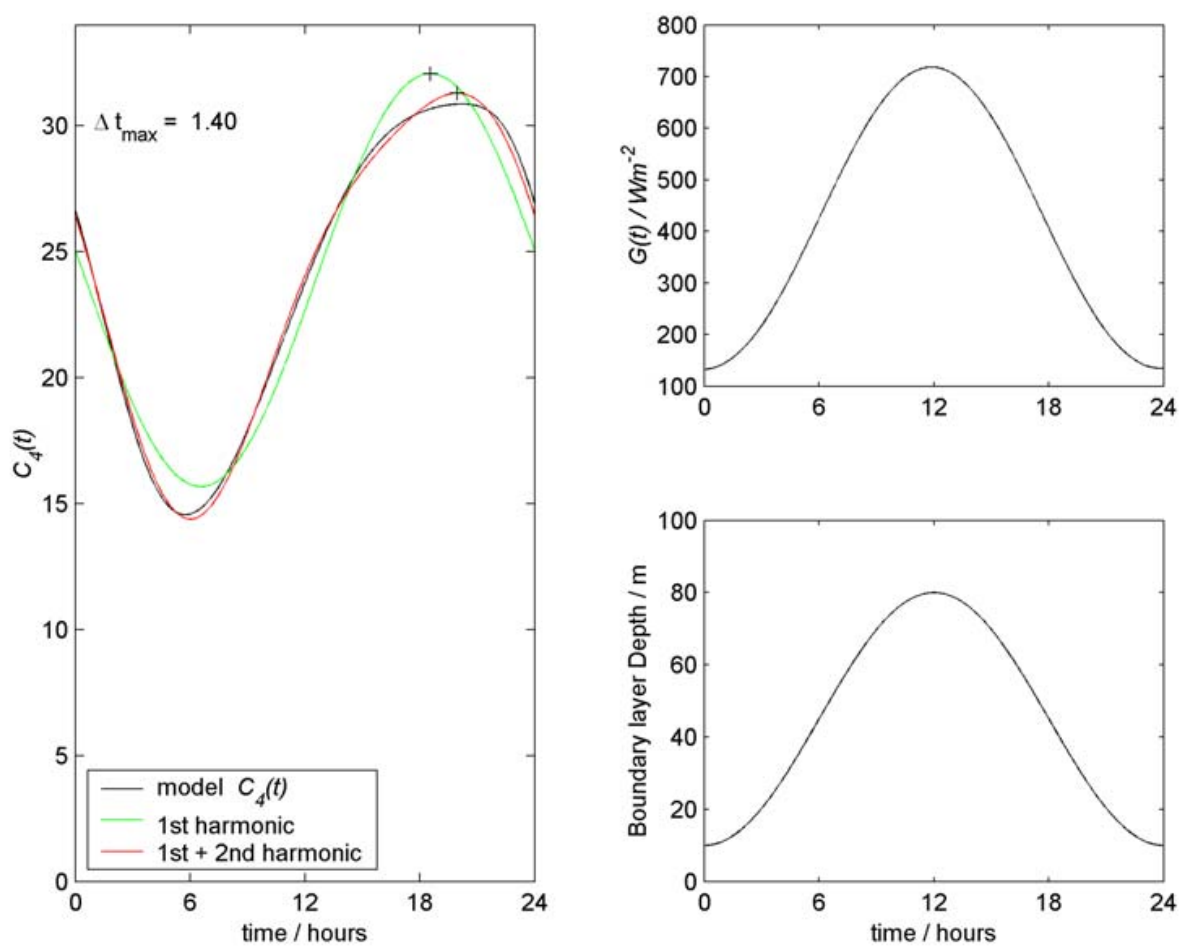

Fig. 8. Four metre tracer concentration time series, $C_{4}(t)$, for a model run where the effective mixing layer depth, $h(t)$, is time dependant. The prescribed surface flux is indicated by $G(t)$, shown in the upper right panel, equivalent to the 30 November at $76^{\circ} \mathrm{S} ; h(t)$ is lower right . Both $G(t)$ and $h(t)$ are symmetric and centred on 12:00 for clarity. $C_{4}(t)$ is asymmetric as indicated by the fitted curves: a single harmonic (green) and $1 \mathrm{st}+2$ nd harmonic (red). The maximum of each curve is shown, the time difference being $1.40 \mathrm{~h}$. Note this figure has been presented to highlight the possible effect of a variable boundary layer depth: the asymmetry in model concentration time series is progressively weaker for dates nearer to winter.

The asymmetry seen in the model output is similar to that observed in the diurnally averaged time series of $\mathrm{NO}_{\mathrm{x}}$ for cloud free days presented in Fig. 2, but the magnitude of the second harmonic is less in the model output than in the $\mathrm{NO}_{x}$ data: the latter has a more distinct ski-jump appearance, with positive curvature during the increasing phase of the curve. The difference between the maxima is remarkably similar; $0.98 \mathrm{~h}$ for the model compared to $1.05 \mathrm{~h}$ for the observed $\mathrm{NO}_{\mathrm{x}}$.

The effect of varying boundary layer depth by an order of magnitude may be only part of the story, but the point being stressed is that simple diffusion, a loss term and boundary layer depth can account for most of the variability observed in an $\mathrm{NO}_{\mathrm{x}}$ time series. The model is admittedly oversimplified, in that there is undoubtedly complex air chemistry of differing time scales involved, and the actual diffusivity profiles are perhaps unrealistic; nevertheless, the visualisation of the twin processes of non-linear diffusion and decay are an aid to a more intuitive understanding of vertical transfer in the boundary layer. Further, when validation studies of the more complete boundary layer chemistry are made, and model and data disagree, the likely parameters causing such disagreement will be easier to track down, study in isolation, and correct given a grasp of the fundamental processes involved.

\section{Conclusions}

A simple boundary layer diffusion model incorporating a loss process is shown to generate trace gas profiles having a number of general characteristics. For the case where there is no transfer out of the top of the boundary layer, and a flux of tracer at the surface, concentration profiles tend to log-linear forms. Time series from the model agree well with field measurements, and differences may be explained by significant variation in the boundary layer depth, itself a result of convection. Model-data agreement does not necessarily identify a process, but does highlight the need to be aware of competing processes within comprehensive boundary layer chemistry model with multiple parameters.

The generalisations from the model help provide an intuitive understanding of the evolution of trace gas species, the form of profiles resulting from surface fluxes, and how tracer measurements can be sensitive to the height of the sampling inlet. 
Acknowledgements. We thank A. Saiz-Lopez for assistance with the uncertainties in air chemistry measurements and diffusion; J. King and A. Weiss for suggested improvements with the text. We also thank the two anonymous reviewers whose comments have improved the clarity of the paper.

Edited by: J. Lee

\section{References}

Ambach, W.: The influence of cloudiness on the net radiation balance of a snow surface with high albedo, J. Glaciol., 13, 73-84, 1974.

Davis, D., Eisele, F., Chen, G., et al.: An overview of ISCAT 2000, Atmos. Environ., 38(32), 5363-5373, 2004.

Dibb, J. E. and Jaffrezo, J. L.: Air-snow exchange investigations at Summit, Greenland: An overview, J. Geophys. Res., 102, 26795-26807, 1997.

Jones, A. E., Anderson, P. S., Wolff, E. W., Turner, J., Rankin, A. M., and Colwell, S. R.: A role for newly forming sea ice in springtime polar tropospheric ozone loss? Observational evidence from Halley station, Antarctica, J. Geophys. Res., 111, D08306, doi:10.1029/2005JD006566, 2006.
King, J. C. and Anderson, P. S.: Heat and Water Vapour fluxes and Scalar Roughness Lengths over an Antarctic Ice Shelf, Bound.Lay. Meteorol., 69, 101-121, 1994.

King, J. C., Argentini, S. A., and Anderson, P. S.: Contrasts between summer time surface energy balance and boundary layer structure at Dome C and Halley stations, Antarctica, J. Geophys. Res., 111, D02105, doi:10.1029/2005JD006130, 2006.

Lee-Taylor, J. and Madronich, S.: Calculation of actinic fluxes with a coupled atmosphere-snow radiative transfer model, J. Geophys. Res., 107(D24), 4796, doi:10.1029/2002JD002084, 2002.

Mahrt, L.: Stratified Atmospheric Boundary Layers, Bound.-Lay. Meteorol., 90, 375-396, 1998.

Warren, S. G.: Optical Properties of Snow, Rev. Geophys. Space Phys., 20, 67-89, 1982.

Trudinger, C. M., Enting, I. G., Etheridge, D. M., Francey, R. J., Levchenko, V. A., Steele, L. P., Raynaud, D., and Arnaud, L.: Modelling air movement and bubble trapping in firn, J. Geophys. Res., 102(D6), 6747-6763, 1997.

Wolff, E. W., Jones, A. E. Martin, T. J., and Grenfell, T. C.: Modelling photochemical $\mathrm{NO}_{\mathrm{x}}$ production and nitrate loss in the upper snowpack of Antarctica. Geophys. Res. Lett., 29(20), 1944, doi:10.1029/2002GL015823, 2002. 